\title{
EXTRAÇÃO DE CONTORNOS DE LESÕES DE PELE UTILIZANDO DIFUSÃO ANISOTRÓPICA E MODELOS DE CONTORNO ATIVO SEM BORDA
}

\author{
Roberta B. Oliveira ${ }^{1}$, Alex F. de Araujo ${ }^{2}$, João Manuel R. S. Tavares ${ }^{2}$, Norian Marranghello ${ }^{1}$, \\ Ricardo B. Rossetti ${ }^{3}$, Aledir S. Pereira ${ }^{1}$
}

${ }^{1}$ Departamento de Ciências de Computação e Estatísticas, Instituto de Biociências, Letras e Ciências Exatas, Universidade Estadual Paulista (correspondente-robyoliveira1 @ gmail.com)

${ }^{2}$ Instituto de Engenharia Mecânica e Gestão Industrial, Faculdade de Engenharia, Universidade do Porto

${ }^{3}$ Clínica DERM, São José do Rio Preto, São Paulo

\begin{abstract}
According to an estimate made by the National Cancer Institute (INCA) in 2012, also valid for the year 2013, the skin cancer appears as one of the most cancer types common in Brazil. The high level of predominance of the skin cancer case has motivated the search and the development of computational methods to assist dermatologists in the diagnosis of skin lesions. The main goal of such methods is concerned to the detection of benign skin lesions to prevent their development, or diagnose malignant lesions at early stages so that they undergo appropriate treatment plans with higher chances of cure. The objective of this paper is to present a computational method for extracting edges of skin lesions from photographic images in order to facilitate the extraction of its main features used for classification. This paper presents a method for the extraction of contours of skin lesions, such as nevi, seborrheic keratosis and melanoma, from images, which uses the technique of anisotropic diffusion to smooth the input images and the active contour model without edges, known as Chan-Vese model, to segment the smoothed image. The application of the anisotropic diffusion filter removes selectively the noise present in the input image. The Chan-Vese model is based on the Mumford-Shah region growth technique, common used in image segmentation tasks, and the Level Set Active Contour model, which allows topological changes of the curves applied on the input images to segment them. Then, a morphological filter is applied on the segmented images in order to eliminate holes in the skin lesion regions and also to smooth their edges. Experimental tests have been accomplished to compare the segmentation results obtained by the traditional thresholding method, by the combination of an anisotropic diffusion model and the Chan-Vese model and by the proposed method using grayscale dermatologic images. This comparison has been revealed that the method proposed is effective to detect skin lesions and extract their contours in dermatologic images.
\end{abstract}

Keywords: Medical Image Analysis, Chan-Vese Model, Anisotropic Diffusion Filter. 


\section{INTRODUÇÃO}

O câncer vem se tornando cada vez mais comum no mundo todo. Aproximadamente 518.510 casos são esperados, de acordo com a estimativa de incidência de câncer no Brasil realizada pelo Instituto Nacional de Câncer (INCA) para o ano de 2012, válidas também para o ano 2013 [18]. Dentre eles podemos citar os cânceres de pele, próstata, pulmão, mama feminina e colo do útero. Sendo os cânceres de pele do tipo não melanoma, tais como os basocelulares e espinocelulares, os mais incidentes na população, correspondendo a $25 \%$ dos casos (134.170 casos novos). O melanoma apesar de ser um dos tipos de câncer menos frequente, representando apenas 4\% dos casos (6.230 casos novos), é o tumor de pele mais agressivo, que apresenta um alto índice de mortalidade [15]. Mas quando diagnosticado precocemente e tratado corretamente, há uma grande chance de cura desse tipo de câncer.

Este grande aumento nos casos de câncer tem motivado a construção de sistemas automatizados para analisar lesões de pele, com o intuito de auxiliar os dermatologistas no diagnóstico de lesões de pele. O objetivo principal é analisar as lesões benignas, para evitar o seu desenvolvimento. Também diagnosticar as lesões malignas em seu estágio inicial, para serem tratadas precocemente, período onde tem mais chances de cura.

Na aplicação destes sistemas são utilizadas imagens de lesões de pele, tiradas dos pacientes pelos próprios dermatologistas. Essas imagens podem ser fotográficas, capturadas por câmeras digitais. As imagens adquiridas podem obter vários ruídos, tais como, reflexos, pêlos, linhas da pele que atrapalham na sua detecção, por isso faz-se necessário realizar uma suavização da imagem. Uma técnica muito utilizada para este fim é a difusão anisotrópica, que permite a suavização seletiva da imagem, ou seja, conforme a quantidade de ruídos presentes na imagem [3, 5 e 13].

A partir das imagens filtradas é possível segmenta-las, ou seja, separar a região doente da saudável, para que possa ser extraído o seu contorno ou suas características a fim de obter informações detalhadas para auxiliar no diagnostico das lesões de pele [ 6 e 21]. A técnica de segmentação introduzida por Chan e Vese [8], é um modelo de contorno ativo sem borda que se baseia na segmentação por região. Esse modelo vem sendo muito utilizado para segmentação de imagem médicas devido as suas diversas vantagens, como a mudança automática da topologia da curva [1, 25 e 26].

O artigo possui a seguinte estrutura: Na seção 2 é abordado técnicas de processamento de imagens para o desenvolvimento do método. Na seção 3 é apresentado o método desenvolvido. Na seção 4 são discutidos os resultados e na seção 5 a conclusão do trabalho.

\section{FUNDAMENTAÇÃO TEORICA}

Nesta seção são abordados os principais conceitos utilizados no trabalho. Esses conceitos envolvem técnicas de processamento de imagens digitais que permitem a filtragem e segmentação de imagens digitais de lesões de pele. 


\subsection{Difusão anisotrópica}

As imagens geralmente são obtidas com ruídos, sendo necessário passarem por uma filtragem. Um filtro não-linear que está sendo muito utilizado para suavizar imagens é a difusão anisotrópica [3, 4, 5 e 9]. Este filtro permite a suavização seletiva da imagem, sendo aplicado por meio de iterações, que são definidas de acordo com a quantidade de ruídos presentes na imagem.

Barcelos, Boaventura e Silva [4] acrescentaram o termo $(1-g)$ da equação de difusão anisotrópica, descrita na Equação 1, para realizar a suavização da imagem com menor perda da borda, com objetivo de aplicá-la em imagens que necessitem de uma maior suavização. Este filtro foi inicialmente proposto por Perona e Malik [24], tendo o seguinte modelo: $u_{t}=\operatorname{div}(g(\nabla u) \nabla u)$. O termo $g|\nabla \mathrm{u}| \operatorname{div}(\nabla \mathrm{u} /|\nabla \mathrm{u}|)$ da Equação 1 foi proposto por Alvarez, Lions e Morel [2], como uma alteração do modelo proposto por Perona e Malik. Já o termo $(u-I)$ adicionado na Equação 1, foi proposto por Nordström [20].

$$
u_{t}=g|\nabla \mathrm{u}| \operatorname{div}\left(\frac{\nabla u}{|\nabla \mathrm{u}|}\right)-\lambda(1-g)(u-I),
$$

onde $u(x, y, 0)=I(x, y)$, sendo $x, y \in \Omega \subset \mathbb{R}^{2} ; u$ é a imagem suavizada; $I$ é a imagem original; $t$ é a escala da suavização, sendo $t>0$; div é o operador divergente; $\nabla u$ é o valor do gradiente de $u ; \lambda$ é um parâmetro que auxilia na velocidade da difusão.

O termo $g$, definido pela Equação 2, é utilizado para detecção de borda. Considerando uma vizinhança de um ponto $x$, quanto o gradiente $\nabla$ possui uma média baixa, ou seja, há poucos pontos (ruídos) na vizinhança, o $x$ é considerado um ponto interior $(g \sim 1)$. Mas caso o gradiente $\nabla$ possua uma média alta, possuindo vários pontos, o $x$ será um ponto de um contorno $(g \sim 0)$.

$$
g=\frac{1}{1+k\left|\nabla\left(G_{\sigma} * u\right)\right|^{2}}
$$

tal que $g(0)=1, g(s) \geq 0$ e $g(s) \rightarrow 0$ quando $s \rightarrow \infty, k$ é uma parâmetro e $G_{\sigma}$ é a função gaussiana, conforme a Equação 3 .

$$
G(x, y)=\frac{1}{2 \pi \sigma^{2}} e^{-\frac{x^{2}+y^{2}}{2 \sigma^{2}}}
$$

Encontrada a borda, o termo $|\nabla \mathrm{u}| \operatorname{div}(\nabla \mathrm{u} /|\nabla \mathrm{u}|)$ da Equação 1 tem por objetivo realizar uma suavização alta em ambos os lados da borda quando $g \sim 1$, e uma suavização baixa na mesma, quando $g \sim 0$. O termo de moderação $(1-g)$ tem função de equilibrar o termo forçante $(u-I)$, juntos reforçam a borda para não perde-la na difusão permitindo uma melhor suavização da imagem. 


\subsection{Modelo de contorno ativo sem borda}

O modelo de contorno ativo sem borda (Chan-Vese), proposto por Chan e Vese [8] para segmentação baseada em região e detecção de objetos em uma imagem. O modelo se baseia na técnica de segmentação Mumford-Shah [19] e também no método Level Set [22], para representar a curva. As principais vantagens desta técnica são:

- A posição da curva inicial pode ser definida em qualquer lugar da imagem;

- Os contornos interiores são automaticamente detectados, sem a necessidade de introduzir uma nova curva na imagem, ao contrário do Level Set;

- Detecção de diferentes objetos com variadas intensidades e ainda com fronteiras borradas;

- Mudança topológica automática da curva;

- Detecção de objetos onde o contorno não possui gradiente. Pois o critério de parada da evolução da curva até a fronteira desejada não depende do gradiente da imagem. Isso não é possível com a utilização do modelo de contorno ativo tradicional.

O modelo Chan-Vese se baseia na segmentação para a minimização de energia. Considerando uma curva $C$, como uma fronteira que separa o domínio da imagem $\Omega$ em dois subconjuntos $\omega$ e $\bar{\omega}$, sendo $\omega$ a região fora da curva $C$ e $\bar{\omega}$ representa a região dentro da curva $C$, onde $\omega \subset \Omega$ e $C=\partial \omega$. Este modelo basicamente considera um termo "apropriado" $F$ de energia funcional (Equação 4) utilizado para a minimização da energia, que faz com que a curva $C$ se deforme em sentido a fronteira $C_{0}$ de uma imagem $u_{0}$ com regiões de intensidades $u_{0}^{i}$ constantes e aproximadas.

$$
F=F_{1}(C)+F_{2}(C),
$$

sendo $F_{1}(C)$ e $F_{2}(C)$ a localização do objeto, se está dentro ou fora da curva $C$, respectivamente. $O$ termo $F$ é calculado por funcionais da seguinte forma:

$$
F=\int_{\text {dentro }(C)}\left|u_{0}(x, y)-c_{1}\right|^{2} d x d y+\int_{\text {fora }(C)}\left|u_{0}(x, y)-c_{2}\right|^{2} d x d y
$$

onde $c_{1}$ é a média da imagem $u_{0}$ dentro da curva $C$ e $c_{2}$ a média fora da curva.

Se o $F_{1}(C)>0$ e $F_{2}(C) \approx 0$, então a curva está localizada fora do objeto. Uma curva se localiza dentro do objeto, quando $F_{1}(C) \approx 0$ e $F_{2}(C)>0$. Nos casos onde a curva se encontra dentro e fora do objeto $F_{1}(C)>0$ e $F_{2}(C)>0$. Outra situação é quando $F_{1}(C) \approx 0$ e $F_{2}(C) \approx 0$, neste caso, o termo de energia $F=0$ está minimizado, ou seja, a curva $C$ está sobre a fronteira $C_{0}$. 


\subsection{Limiarização}

A técnica de limiarização (thresholding) pode ser utilizada na etapa de segmentação para separar os objetos do fundo da imagem. Esse processo tem como resultado uma imagem binária, onde a cor preta pode representar o objeto e a branca o fundo. A sua aplicação envolve a escolha de um limiar $(T)$ para fazer a separação dos níveis de cinza da imagem, que são representados pelo histograma ${ }^{*}$. Este limiar pode ser representado apenas por um valor (limiar único) ou corresponder a um intervalo (limiares múltiplos).

No caso da seleção de um limiar, a aplicação da limiarização segue a seguinte condição [14 e 16]:

$$
\begin{aligned}
& g(x, y)=1 \text { se } f(x, y) \geq T \\
& g(x, y)=0 \text { se } f(x, y)<T
\end{aligned}
$$

onde $g(x, y)$ representa cada ponto da imagem de saída, que receberá 1 quando o pixel da imagem original $f$ nas coordenadas $(x, y)$ for maior ou igual ao limiar $T$ ou 0 quando a intensidade do pixel for menor que o limiar. Os pixels com valores transformados para 1, ou seja, cor branca, representam o fundo e os definidos com 0 , estabelecem o objeto da imagem com cor preta. Também podem ser escolhidos vários limiares, determinando intervalos e condições diferentes de limiarização. Podendo ser estabelecido da seguinte forma: os pixels da imagem $f(x, y)$ com intensidade entre os valores do intervalo definidos pelos limiares $T_{1}$ e $T_{2}$, sendo $T_{1}<f(x, y) \leq T_{2}$, recebem o valor 0 , sendo considerado um objeto e os pixels maiores que $T_{2}$, ou seja, $f(x, y) \leq T_{1}$, recebem o valor 1 , representando o fundo da imagem e no caso dos valores menores ou iguais a $T$, tal como, $\mathrm{f}(x, y) \leq T_{2}$, recebem um nível de cinza diferente, representando outro objeto.

O limiar é definido por uma função, que pode utilizar a intensidade original do pixel, sendo considerado um limiar global ou seu valor pode ser adquirido por alguma propriedade, como a média dos seus vizinhos, neste caso o limiar é considerado local. Um exemplo de limiar utilizado na binarização das imagens é o método de OTSU.

O método de OTSU [23] se baseia no histograma normalizado da imagem e considera a distribuição de probabilidade $P_{i}$ em uma imagem com $L$ níveis de cinza $[1,2, \ldots, L]$, conforme a Equação 8.

$$
P_{i}=\frac{n_{i}}{N}
$$

onde $n_{i}$ representa o número de pixels no determinado nível de cinza $i$ e $N$ é o número total de pixels da imagem, sendo $N=n_{1}+n_{2}+\cdots+n_{L}$.

\footnotetext{
${ }^{*}$ Representação gráfica da quantidade de pixel para cada nível de cinza da imagem.
} 


\subsection{Filtros morfológicos}

A morfologia matemática é baseada na teoria de conjuntos e são aplicadas em diversos trabalhos na área de processamento de imagens [6, 7 e 21]. Utilizada para realce, filtragem, segmentação, detecção de borda, dentre outras atividades. Consiste na extração ou modificações das informações referentes a estrutura geométrica dos objetos presentes na imagem por meio de uma elemento estruturante definido. $\mathrm{O}$ elemento estruturante representa uma máscara, com determinado tamanho, podendo ser estruturada com diferentes formas, tais como, quadrada, circular, elíptica e cruz.

As operações de abertura e fechamento são utilizadas para formar filtros morfológicos $(A \circ B) \cdot B$, com objetivo de remover ruídos isolados em uma imagem, que sejam menores que o elemento estruturante.

\section{Abertura}

A operação de abertura de um conjunto $A$ (imagem) e $B$ (elemento estruturante) é aplicada utilizando a seguinte definição:

$$
A \circ B=(A \ominus B) \oplus B
$$

O resultado desta operação é representado por $A \circ B$, sendo realizada a operação de erosão $A \ominus B$ entre $A$ e $B$, e logo após se aplica a operação de dilatação. A abertura suaviza os contornos da imagem, elimina pequenos pontos por meio da erosão, tendo sua forma recuperada pela dilatação e também separa regiões. Após a abertura a região fica bem parecida com a original, mas possui menos detalhes e tende a abrir pequenos vazios na imagem.

\section{Fechamento}

Essa operação é o inverso da abertura, ou seja, o conjunto $A$ primeiro é dilatado pelo elemento estruturante $B$ e depois é feita a erosão. A operação de fechamento é dada por:

$$
A \cdot B=(A \oplus B) \ominus B
$$

O objetivo principal desta operação é estabelecer um conjunto sem modificar o seu tamanho e forma inicial. O elemento estruturante possibilita preencher os buracos menores do que o mesmo, juntar regiões próximas, além de suavizar as bordas, mas o conjunto fechado não é tão detalhado quanto o conjunto inicial.

\section{MÉTODO DESENVOLVIDO}

Neste capítulo apresenta-se o desenvolvimento do método proposto para análise de lesões de pele, com intuito de auxiliar o dermatologista no seu diagnóstico. Na Figura 1, temos o diagrama de fluxo do método desenvolvido. Este método é baseado nas etapas de proces- 
samento de imagens digitais. Sendo dividida da seguinte maneira: aquisição de imagens para formar o banco, pré-processamento, segmentação e pós-processamento, tendo como resultado final o contorno da lesão.

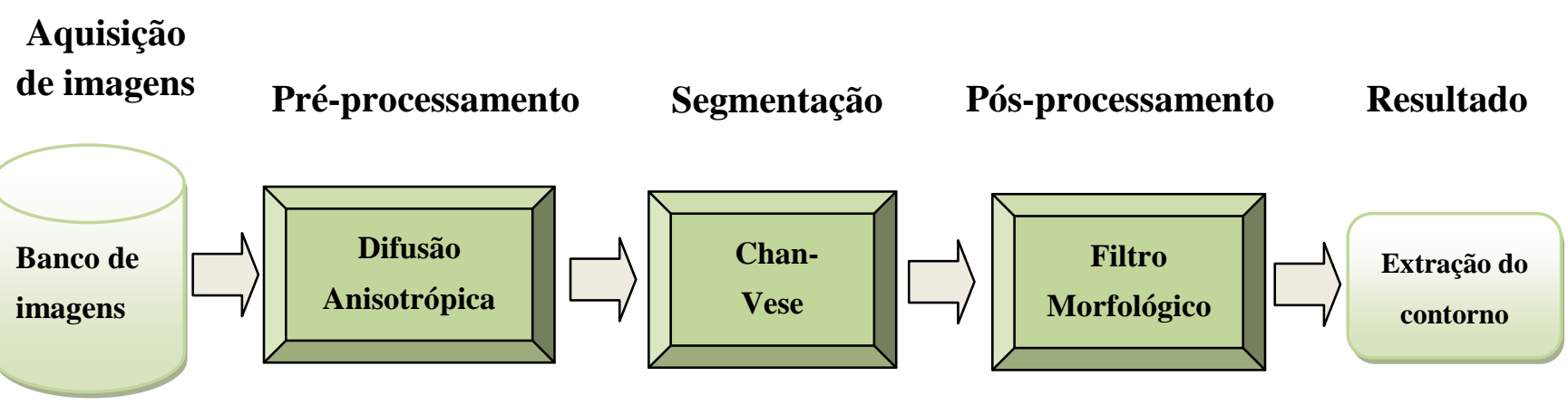

Figura 1. Diagrama de fluxo do método desenvolvido.

As imagens do banco passam por um pré-processamento que utiliza o filtro de difusão anisotrópica para eliminar os ruídos provenientes da etapa de aquisição de imagens. Depois é realizada a segmentação, utilizando o modelo de contorno ativo sem borda Chan-Vese. Para eliminar ruídos, orifícios e suavizar a borda nas imagens resultantes do processo de segmentação, são utilizados filtros morfológicos. A aplicação desses métodos possibilita a extração do contorno.

\subsection{Aquisição de imagens}

A primeira etapa para o desenvolvimento do método proposto foi a aquisição de imagens para a formação de uma base de imagens de lesões de pele, utilizada para os testes. A base deste trabalho foi formada por imagens dos seguintes bancos de imagem: Loyola University Chicago [17], YSP Dermatology Image Database [12], 10 [10] e DermIS [11].

A base é constituída de 408 imagens, sendo 62 de nevos melanocíticos, 84 de Ceratose Seborréica e 260 de Melanoma. As imagens utilizadas nos testes foram convertidas de JPG para BMP com 16 bits e para dimensão 200 x 200, para facilitar o processamento das mesmas. Sendo composta por imagens adquiridas de câmeras fotográficas, do tipo JPG, com dimensões variadas, diferentes aproximações e diversas influências de ruído, tais como, reflexo, linhas da pele e pêlos.

\subsection{Pré-processamento}

Nesta etapa foi realizada a suavização nas imagens da base, com o intuito de amenizar os efeitos dos ruídos presentes, tais como, pêlos, reflexos e linhas da pele, que podem influenciar o resultado da segmentação. Foi utilizado o filtro de difusão anisotrópica, proposto por Barcelos, Boaventura e Silva [4]. 
A implementação deste filtro foi baseada na Equação 1, que possui os seguintes parâmetros: $\Delta t$ determina o tamanho da evolução temporal em que cada iteração da difusão será realizada; o parâmetro $\sigma$ é o desvio padrão da função Gaussiana $G_{\sigma}$; o parâmetro $\lambda$ ajuda a reforçar a borda; $k$ auxilia a função gaussiana a definir se o ponto faz parte da borda ou não, caso seja um ponto da borda, este sofrerá menos suavização; e a imagem será suavizada, de acordo com o número de iterações.

Os parâmetros são fixados com os seguintes valores: $\Delta t=0.1, \sigma=1, \lambda=1, k=$ 0.0008 e 100 iterações. O resultado da aplicação deste filtro pode ser visto na Figura 2.

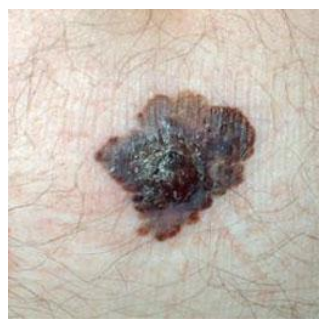

(a)

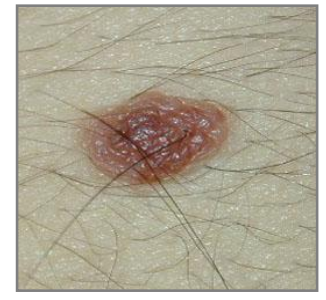

(c)

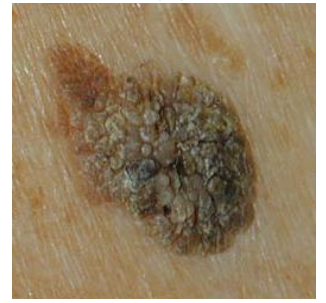

(e)

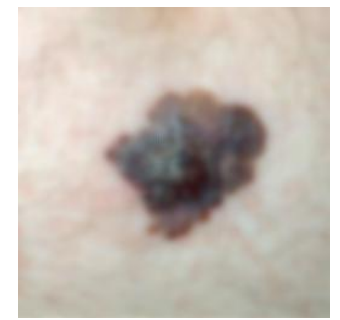

(b)

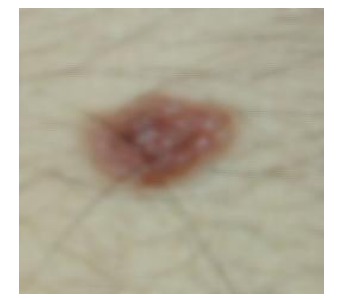

(d)

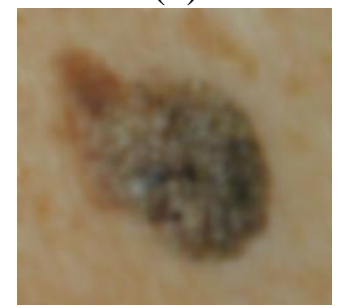

(f)

Figura 2: Resultado da aplicação do filtro de Difusão anisotrópica. As imagens em (b), (d) e (f) são resultados da aplicação do filtro nas imagens originais em (a), (c) e (e), respectivamente.

Na Figura 2, temos três imagens originais em (a), (c) e (e), representando cada tipo das lesões de pele abordadas neste trabalho (melanoma, nevo melanocítico e ceratose seborréica). Em (b), (d) e (f) temos as imagens suavizadas pelo filtro de Difusão anisotrópica. Podemos observar que o filtro amenizou os efeitos ruídos, tais como, pêlos e linhas da pele, sem eliminar o contorno da lesão.

\subsection{Segmentação}

A segmentação das imagens de lesões de pele consiste em separar a região doente da região saudável, as tornando homogenias, para que as lesões possam ser localizadas e se con- 
torno extraído. Antes desta etapa foi realizada a suavização das imagens para minimizar os efeitos de ruídos.

A técnica utilizada para a segmentação das imagens neste trabalho é o modelo de contorno ativo sem borda, proposto por Chan e Vese [8]. São várias as vantagens deste método, que permite que seu uso tenha bons resultados, como a mudança automática da topológia da curva.

Primeiramente para a aplicação deste modelo a imagem suavizada em RGB é transformada em níveis de cinza e então definida uma curva sobre a mesma. A curva é posicionada próxima ao centro da imagem, desta forma são realizadas menos iterações para a curva envolver completamente a lesão. A forma inicial da curva foi definida como quadrada, com dimensão $140 \times 140$. No caso das imagens onde a captura da lesão foi mais distante, tornando-as menores, foi definida uma curva $40 \times 40$.

Para a evolução da curva, foram definidos os seguintes parâmetros fixos: $\mu=0.2$, parâmetro que controla o comprimento da curva; $v=0$, influência na área dentro da curva; $\lambda_{1} \mathrm{e}$ $\lambda_{2}=1$, auxilia na localização do objeto dentro e fora da curva respectivamente; $h=1$, auxilia na detecção do contorno interior; $\Delta t=0.1$, é tempo da evolução.

Foram definidas 500 iterações para a evolução da curva, ou seja, a minimização da mesma ocorrerá até o número de iterações ou quando a curva $C$ estiver localizada sobre o objeto. O resultado da aplicação do modelo Chan-Vese possibilita a binarização da imagem, como pode ser visto na Figura 3.
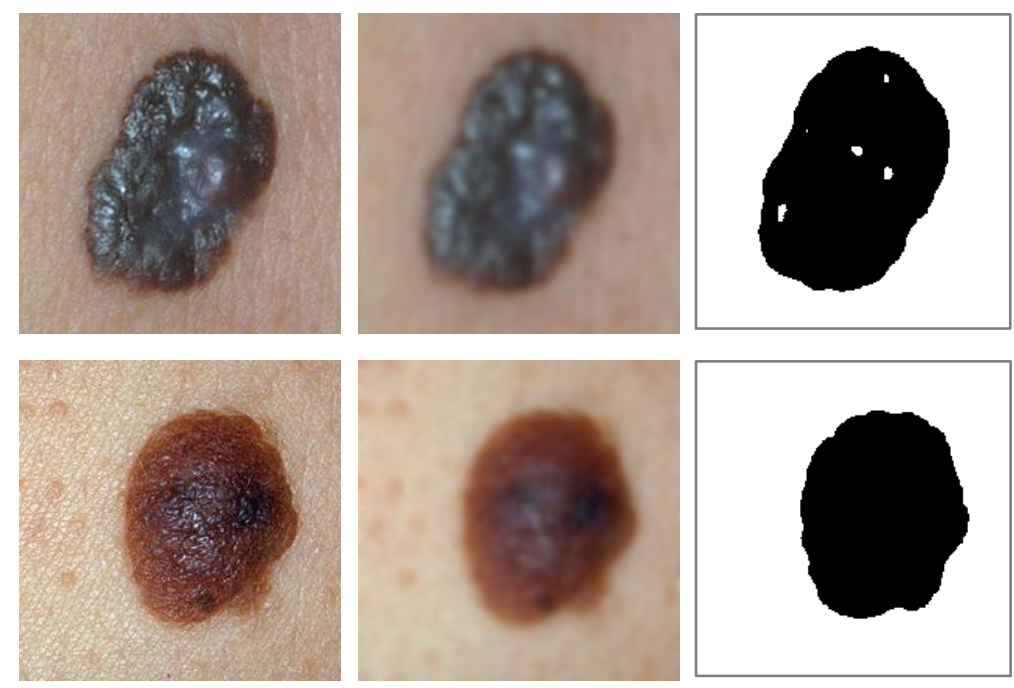

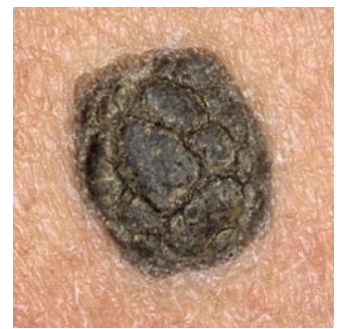

(a)

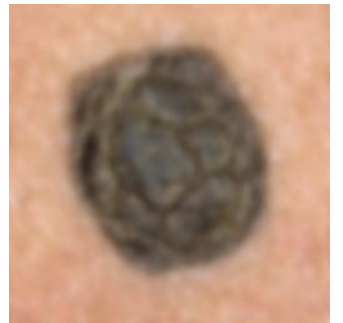

(b)

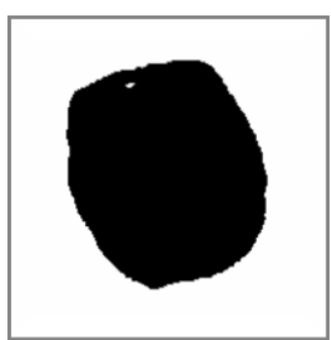

(c)

Figura 3: Aplicação do modelo Chan-Vese. Em (a) temos a imagem original, (b) a imagem suavizada com difusão anisotrópica e (c) segmentação Chan-Vese. 


\subsection{Pós-processamento}

Depois de feita a segmentação é aplicado um filtro morfológico nas imagens binarizadas para tratá-las, eliminado ruídos dentro e fora das regiões segmentadas, devido a definição de bordas falsas pelo método de segmentação, como os reflexos.

O filtro utilizado neste trabalho foi a abertura seguida do fechamento, utilizando um elemento estruturante em forma de elipse, com raio quatro, com o intuito de suavizar a borda, além de eliminar os ruídos. Na Figura 4(c) pode ser visto o resultado da aplicação deste filtro morfológico, nas imagens segmentadas pelo modelo de contorno ativo Chan-Vese da Figura 4(b).
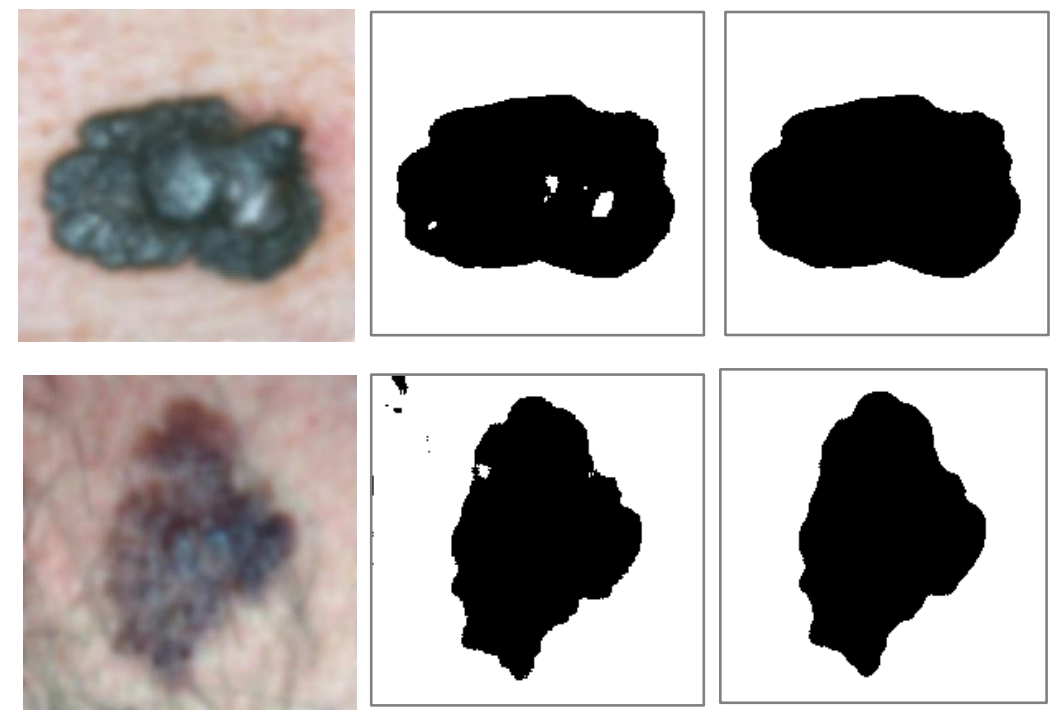

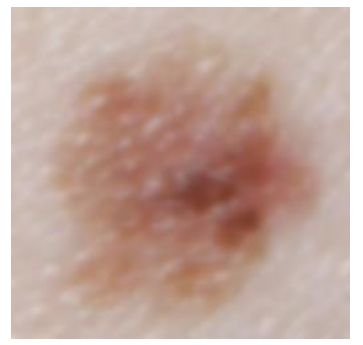

(a)

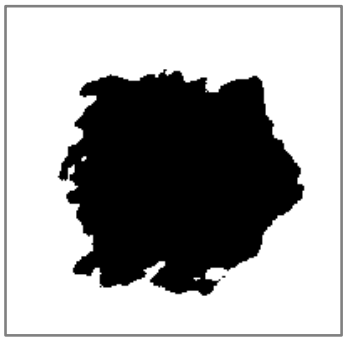

(b)

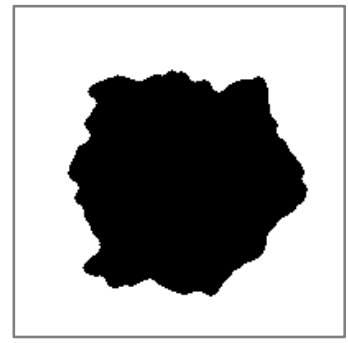

(c)

Figura 4: Aplicação do filtro morfológico de abertura seguido de fechamento. Em (a) imagem suavizada, (b) imagem segmentada e (c) imagem depois de aplicado o filtro morfológico.

Nas imagens resultantes da aplicação do filtro morfológicos apresentadas na Figura 4(c), podemos observar que os orifícios e pontos isolados da região da lesão foram eliminados, além de suavizar a borda sem compromete-lá. 


\subsection{Extração da lesão}

Depois de realizada a última etapa de processamento das imagens de lesão de pele, descrita a cima, o seu contorno é definido e a lesão extraída da pele. O contorno representa as delimitações e irregularidades da borda e a extração da lesão, ou seja, a separação da região doente da região saudável é importante para que suas características possam ser adquiridas, sem influência da pele.

Na Figura 5 pode ser visto o resultado do contorno (b), a definição da borda na lesão (c) e a sua extração (d) conforme a imagem binarizada depois da aplicação do filtro morfológico (a).

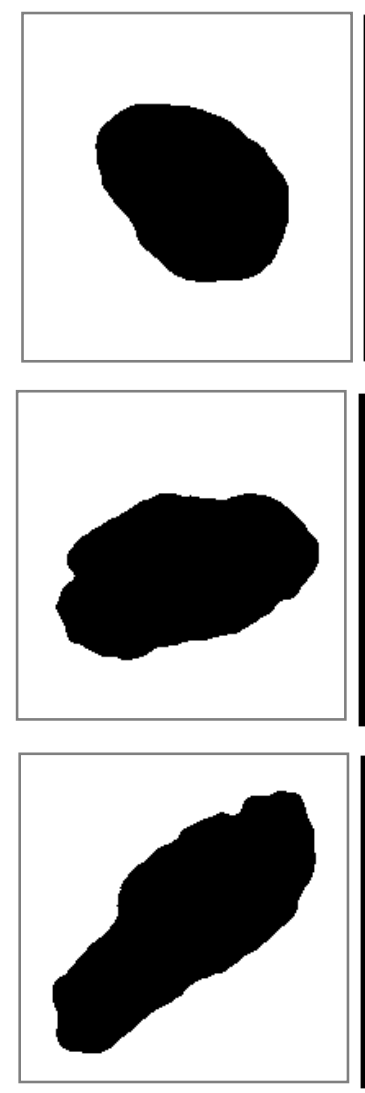

(a)
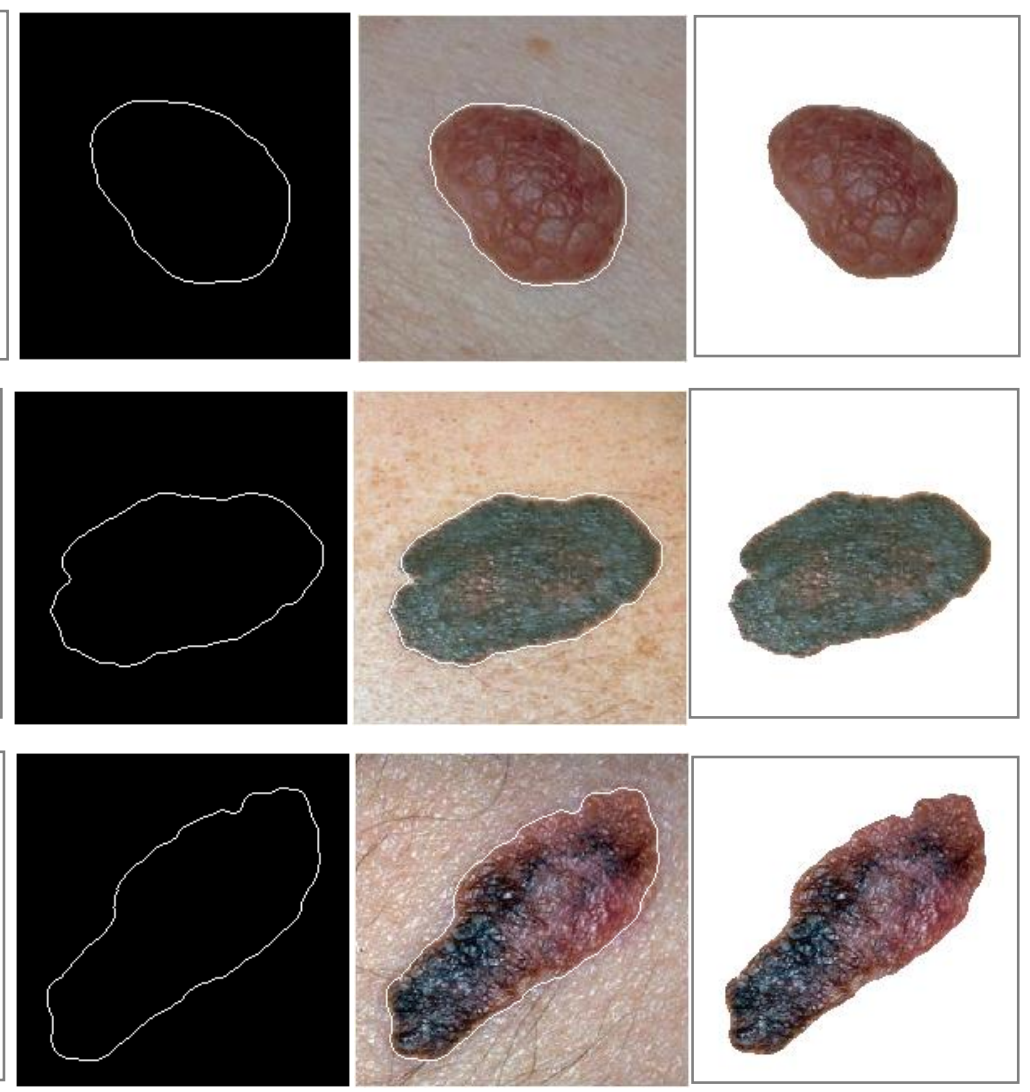

(b)

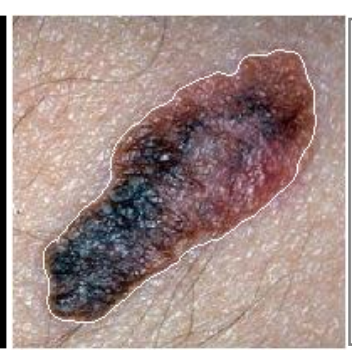

(c)

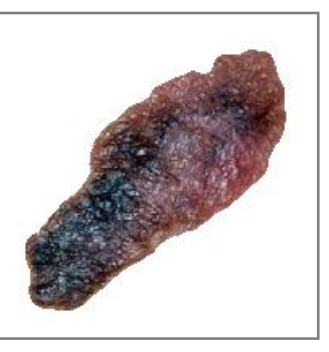

(d)

Figura 5: Definição do contorno e extração da lesão de pele. Na imagem da figura (a) temos o resultado depois de aplicado o filtro morfológico, em (b) a representação do seu contorno, em (c) o contorno sobre a lesão e em (d) a extração da lesão.

O contorno é estabelecido nos pixels onde ocorrem as mudanças bruscas de intensidade na imagem binarizada. Considerando que as lesões são de cor preta e a pele de cor branca, como determinadas neste trabalho, ao percorrer a imagem pixel a pixel, quando houver a mudança de cor, o pixel na mesma posição na imagem original recebe a cor branca para definir a borda. A extração da lesão apresenta somente a região doente conforme a binarização, representada pela cor preta e o restante da imagem, ou seja, a pele saudável é formada com a cor branca. 


\section{RESULTADOS E DISCUSÃO}

Os testes realizados comparam duas técnicas de segmentação para a detecção de lesões de pele: limiarização e o modelo Chan-Vese. As duas técnicas foram aplicadas nas imagens suavizadas pela Difusão anisotrópica. Em seguida foram definidos os contornos das lesões a partir das imagens pós-processadas pelos filtros morfológicos. Esta etapa elimina os orifícios dentro da região que representa a lesão, os ruídos externos e suaviza o contorno.

Método A: a segmentação da imagem é realizada pela técnica de Limiarização. pelo limiar de OTSU. As intensidades menores que o limiar são transformadas em zero, para representar a lesão. Já as intensidades maiores que o limiar recebem um, representando a região saudável.

Método B: realiza a segmentação utilizando o modelo Chan-Vese, utilizando os seguintes parâmetros para a evolução da curva: $\mu=0.2, v=0, \lambda_{1}$ e $\lambda_{2}=1, h=1, \Delta \mathrm{t}=0.1 \mathrm{e}$ 500 iterações.

Podemos visualizar na Figura 6 o resultado da aplicação dos dois métodos de segmentação na imagem original (a). O resultado do Método A é apresentado na imagem (b) e a aplicação do Método B pode ser vista na imagem (c). Podemos observar que o Método B, que utiliza o modelo Chan-Vese para segmentação, obteve melhor resultado do que o Método A, de Limiarização, pois o contorno envolveu melhor a lesão. No resultante do método A, algumas regiões da lesão não foram detectadas corretamente, como pode ser visto na imagem (b).

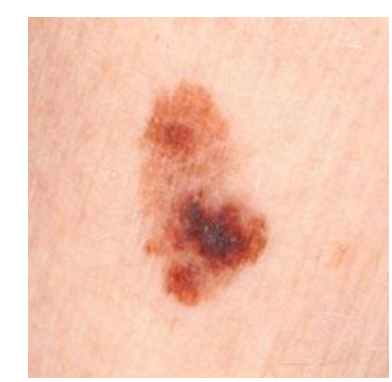

(a)

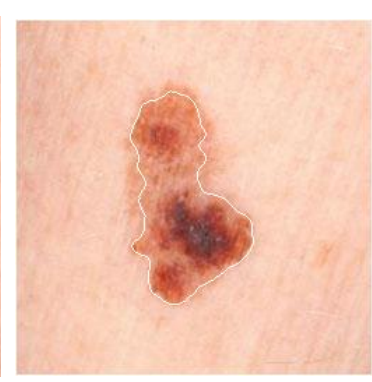

(b)

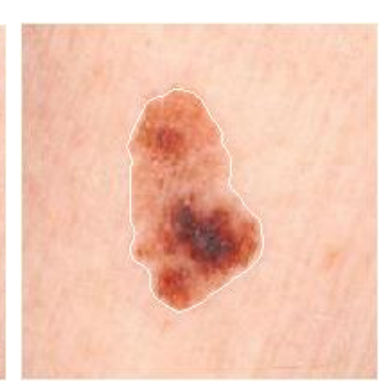

(c)

Figura 6: Resultado da comparação entre métodos de segmentação. Em (a) a imagem original, com aplicação da técnica de limiarização (b) e em (c) a segmentação utilizando o modelo Chan-Vese.

Na Tabela 1 são vistos outros resultados entre os métodos A e B, onde o método B proporciona melhores resultados, que motivaram sua utilização do desenvolvimento deste trabalho. Na primeira coluna é especificado um número para representar as imagens originais da segunda coluna que foram processadas e na terceira e quarta colunas são apresentados os resultados do Método A e B, respectivamente. 
Tabela 1: Resultados obtidos entre dois métodos diferentes de segmentação.
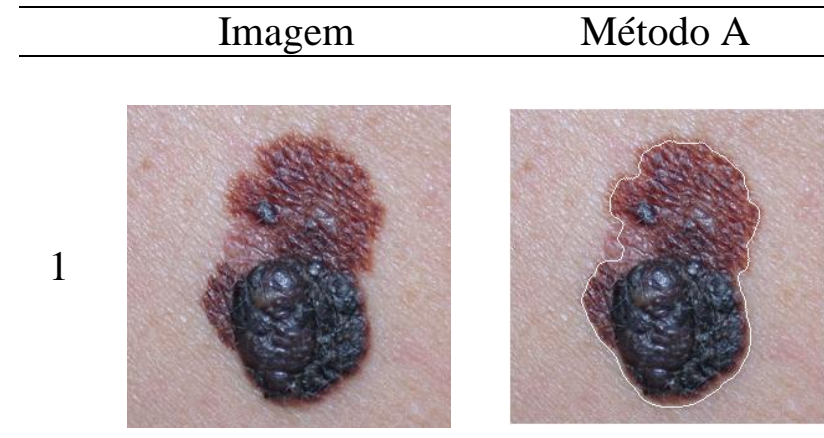

Método B
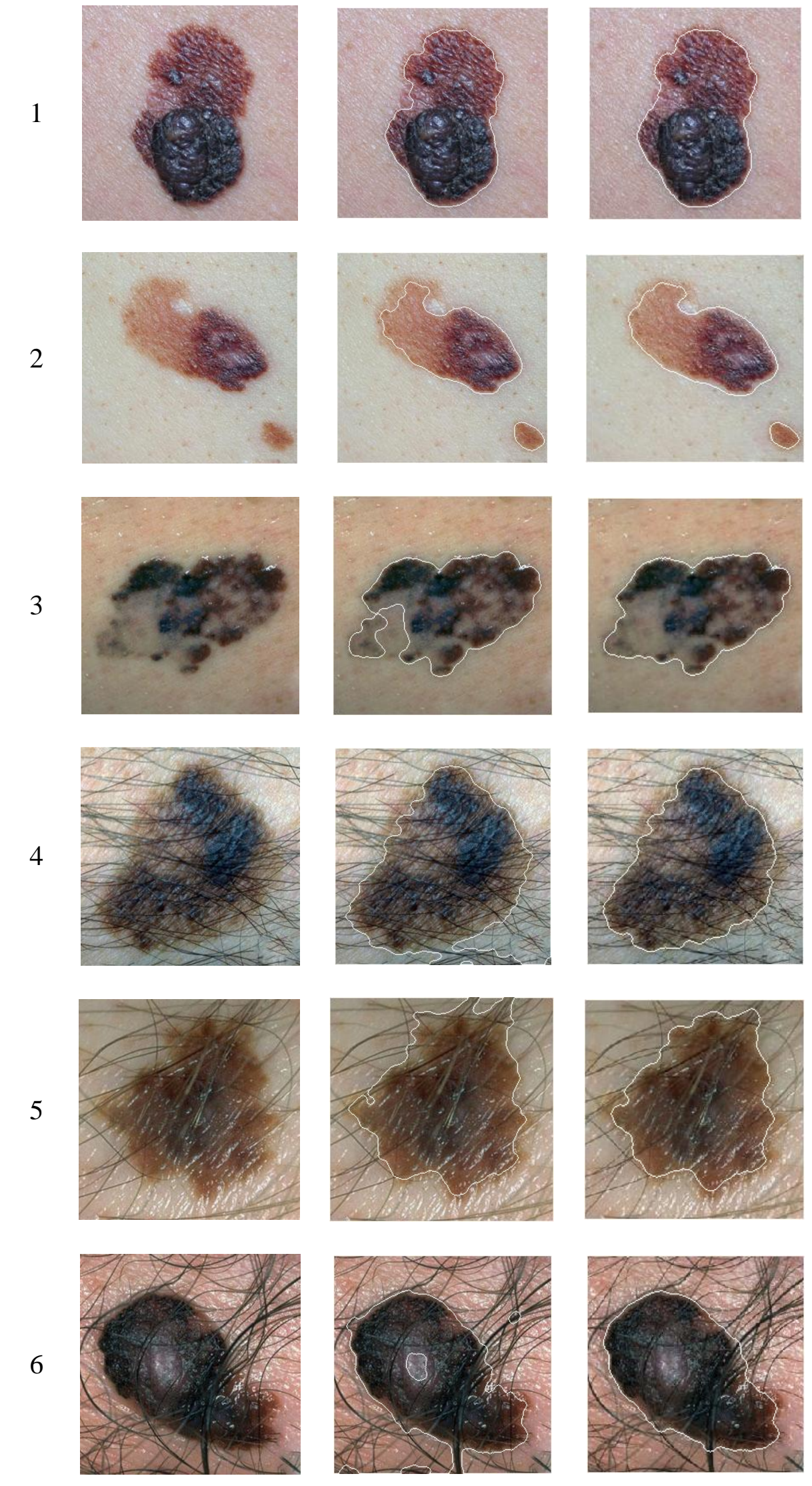


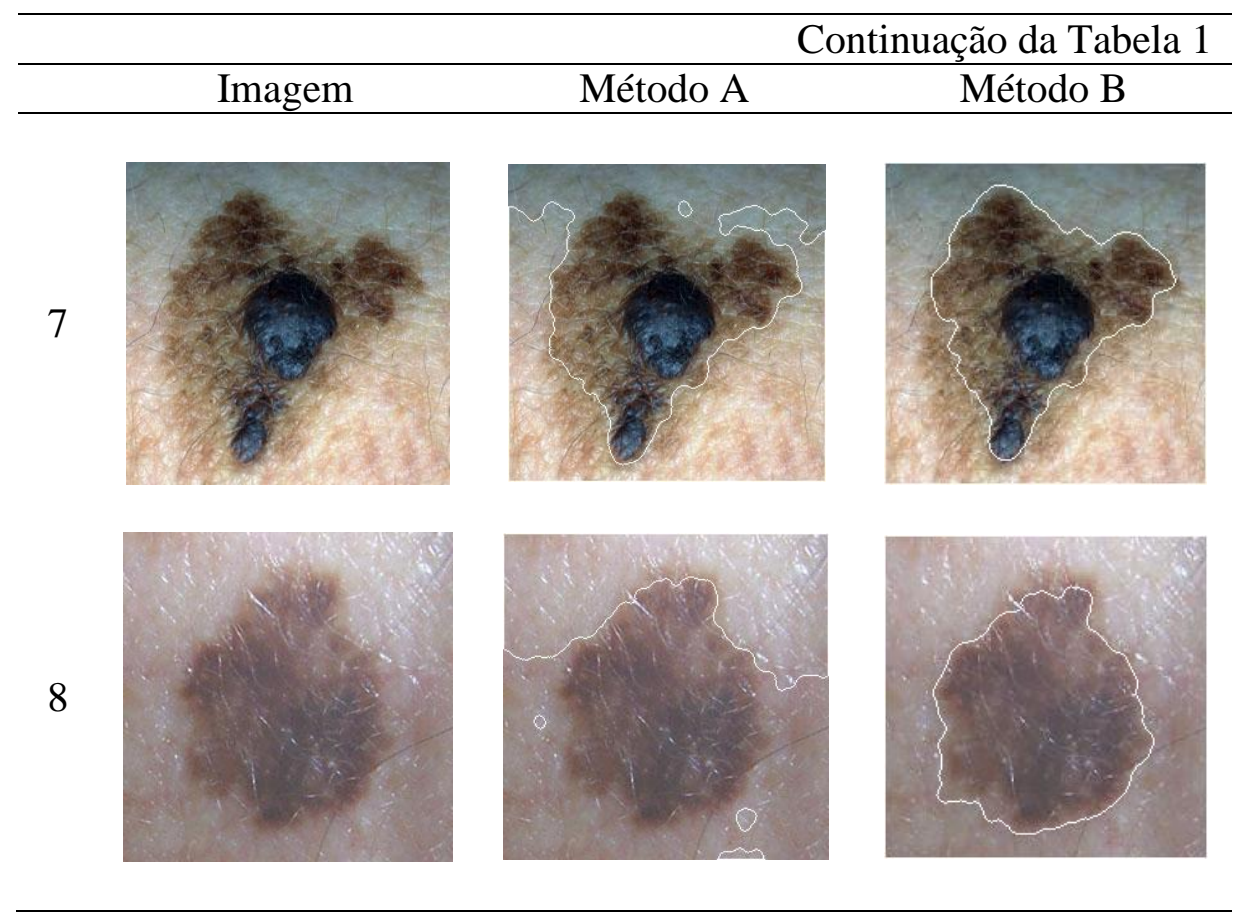

Os resultados do Método B das imagens de 1 a 3 da Tabela 1 obtiveram melhor envolvimento das regiões com intensidades mais próximas da cor da pele do que o Método A, apesar de não envolver totalmente as regiões como na imagem 1 e 2 . No caso das imagens muito ruidosas, o Método B também alcançou melhores resultados, com a influência do filtro de Difusão anisotrópica, como pode ser visto nas imagens 4 a 6 , tendo a lesão detectada com pouca influência de ruído. Já as imagens aplicadas o Método A, alguns pêlos foram detectados e também conectados a lesão. As imagem 7 e 8 possuem a influência de sombra e reflexo, que foram encontrados pelo Método A e já o Método B detectou somente a lesão, apesar da região doente não ter sido envolvida totalmente.

A aplicação do modelo de contorno ativo sem borda (Chan-Vese) obteve uma boa detecção das lesões de pele, como pode ser visto na Tabela 2, o resultado da analise visual.

Tabela 2. Resultado da aplicação modelo Chan-Vese

\begin{tabular}{ccc}
\hline Lesões & $\begin{array}{c}\text { Lesões } \\
\text { detectadas }\end{array}$ & $\begin{array}{c}\text { Lesões não } \\
\text { detectadas }\end{array}$ \\
\hline Melanoma & $98,07 \%$ & $1,93 \%$ \\
Nevo melanocítico & $94,19 \%$ & $5,81 \%$ \\
Ceratose seborréica & $93,55 \%$ & $6,45 \%$ \\
\hline
\end{tabular}

De acordo com os resultados mostrados acima, o método desenvolvido se mostrou animador para detecção das lesões de pele e extração do seu contorno, com o objetivo de auxiliar o dermatologista em seu diagnóstico. 


\section{CONCLUSÕES}

Neste trabalho foi apresentado um método de extração de contornos de lesões de pele. Para minimizar os efeitos dos ruídos foi utilizando o filtro não linear de difusão anisotrópica. O modelo de Contorno ativo sem borda foi aplicado com o intuito de estabelecer uma melhor borda para a lesão, pois utiliza o Mumford-Shah, considerado uma ótima técnica de segmentação por crescimento de região e o Level Set, um modelo de contorno ativo que possibilita mudanças topológicas.

Com o filtro de difusão anisotrópica realizou-se a suavização das imagens sem perder as informações da borda, além de permitir uma suavização seletiva das imagens. Foi eliminado parcialmente a influencia dos ruídos, permitindo uma melhor segmentação das imagens muito ruidosas.

O modelo Chan-Vese se mostrou uma animadora técnica para segmentação de lesões de pele. Apesar de não conseguir localizar corretamente as bordas de algumas imagens quando possuem uma grande área de reflexo ou sombra na parte externa da lesão, pois estas áreas foram consideradas pertencentes a ela. Outro problema são os reflexos interiores muito grandes, não permitindo sua eliminação pelos filtros morfológicos, considerando que o elemento estruturante não pode ser muito grande para não prejudicar a irregularidade da borda. Isso fez com que algumas imagens fossem estabelecidas bordas internas falsas.

A definição do contorno também foi prejudicada nos casos onde as fronteiras das lesões continham reflexos, sombras e intensidades muito claras. No caso das imagens com muitos pêlos, os mesmos puderam influenciar na detecção da lesão, mesmo tendo como característica a detecção dos objetos em imagens ruidosas, mas com a utilização do filtro de difusão anisotrópica os resultados são animadores, exceto nas partes com muitos cabelos. Sendo assim, os reflexos são uns dos principais atributos para segmentação não ser eficiente. Para resolver este problema, é necessária uma captura correta da imagem.

Devido a diferença de tamanho das lesões na imagem, ou seja, conforme sua proximidade no momento da aquisição, a definição da curva na imagem para a aplicação do modelo Chan-Vese, estabelece a precisão da detecção da borda da lesão. Apesar de uma das principais características deste modelo ser a definição do contorno em qualquer região da imagem, quanto mais próxima ela estiver da lesão, mais terá um bom resultado, pois além de detectar a lesão em menos tempo, com menos iterações, é menor a possibilidade de encontrar bordas falsas, como as causadas por reflexos. Desta forma, é necessária uma curva maior para as lesões grandes e uma menor para as lesões pequenas. Por esse motivo a quantidade de iterações às vezes não satisfaz todos os casos, ou seja, se o número de iterações for muito pequeno, devido as imagens grandes ser processadas mais rápidas, as imagens pequenas não conseguem ser totalmente envolvidas no mesmo tempo. Já com um número de iterações alto, a curva passa a procurar por outras fronteiras, podendo envolver objetos falsos. Uma solução seria definir uma curva próxima da lesão e com uma dimensão aproximada para cada tamanho diferente, assim o método detectaria as bordas das imagens grandes e pequenas, aproximadamente ao mesmo tempo.

A utilização do filtro morfológico para tratar a imagem, com ruídos externos, orifícios interiores e bordas muito serrilhadas, obtidas pelo método de segmentação, permitiu uma me- 
lhor definição da lesão, exceto em casos onde a área de reflexo é maior que seu elemento estruturante.

O método desenvolvido neste trabalho permite uma boa detecção e extração dos contornos das lesões de pele, considerando os resultados apresentados da análise visual, sendo 98,07\% de detecção das lesões para as imagens do tipo melanoma, 94,19\% para as imagens de nevos melanocíticos e $93,55 \%$ de detecção das lesões de ceratose seborréica. Com o aprimoramento destas técnicas utilizadas, para solucionar os problemas encontrados nos testes, o método proposto poderá ser muito interessante na detecção das lesões de pele para que possam ser extraídas suas informações e serem passadas ao dermatologista no intuito de auxiliálo no diagnóstico.

\section{Agradecimentos}

Os autores agradecem a Coordenação de Aperfeiçoamento de Pessoal de Nível Superior CAPES, ao Conselho Nacional de Desenvolvimento Científico e Tecnologia - CNPq e a Fundação para a Ciência e Tecnologia - FCT.

\section{REFERÊNCIAS}

[1] Abbas, Q.; Fondón, I.; Rashid, M. "Unsupervised skin lesions border detection via twodimensional image analysis". Computer Methods and Programs in Biomedicine, COMM3090, p. 1-15, 2010.

[2] Alvarez, L.; Lions, P.-L.; Morel, J.-M. "Image Selective Smoothing and Edge Detection by Nonlinear Diffusion". SIAM Journal on Numerical Analysis, v. 29, n. 3, p. 845-866, 1992.

[3] Araujo, A. F. "Método para extração e caracterização de lesões de pele usando difusão anisotrópica, crescimento de regiões, watersheds e contornos ativos". Dissertação de Mestrado em Ciência da computação - Instituto de Biociência, Letras e Ciências Exatas, Universidade Estadual Paulista, São José do Rio Preto, 2010.

[4] Barcelos, C. A. Z.; Boaventura, M.; Silva Junior, E. C. "A well-balanced flow equation for noise removal and edge detection". IEEE Transactions on Image Processing, v. 12, n. 7, p. 751-763, 2003.

[5] Barcelos, C. A. Z.; Pires, V. B. "Na automatic based nonlinear diffusion equations scheme for skin lesion segmentation". Applied Mathematics and Computation, v. 215, p. 251-261, 2009.

[6] Beuren, A. T.; Pinheiro, R. J. G.; Facon, J. "Abordagem morfológica de segmentação do melanoma”. In: Workshop de Visão Computacional, 7. Curitiba, 2011, p. 249-254. 
[7] Celebi, M. E.; Kingravi, H. A.; Iyatomi, H.; Aslandogan, Y. A.; Stoecker, W. V.; Moss, R. H.; Malters, J. M.; Grichnik, J. M.; Marghoob, A. A.; Rabinovitz, H. S.; Menzies, S. W. "Border detection in dermoscopy images using Statistical Region Merging". Skin Research and Technology, v. 14, p. 347-354, 2008.

[8] Chan, T. F.; Vese, L. A. "Active contours without edges". IEEE Transactions on Image Processing, v. 10, n. 2, p. 266-277, 2001.

[9] Coser, L. "Filtro de difusão anisotrópica orientado por evidência de borda". Dissertação de Mestrado em Ciência da computação - Faculdade de Engenharia Elétrica, Universidade Federal de Santa Catarina, Florianópolis, 2009.

[10] Dermatlas. Cohen, B. A.; Lehmann, C. U. Johns Hopkins University - DermAtlas. Disponível em Dermatology Image Atlas: <http://dermatlas.med.jhmi.edu/> Acesso em: 2012.

[11] Dermis. Diepgen TL, Yihune G et al. Dermatology Information System - DermIS. Disponível em Atlas Dermatológico Online: <http://www.dermis.net/dermisroot/en/home/index.htm>. Acesso em: 2012.

[12] Dermatology database. Y. Suzumnura. YSP Dermatology Image Database - Japan. Disponível em YSP Dermatology Image Database: <http://homepage1.nifty.com/ysh/indexe.html>. Acesso: em 2012.

[13] Dong, G.; Palaniappan, K. Benchmarking. "A robust method for edge-preserving image smoothing”. Lecture Notes in Computer Science, v. 5259, p. 390-399, 2008.

[14] Gonzalez, R. C.; Woods, R. E. "Digital image processing”. 2. ed. New Jersey: Prentice Hall, 2002. 793 p.

[15] INCA. Instituto Nacional De Câncer. "Tipos de câncer: Pele melanoma e pele não melanoma”. Disponível em: < http://www2.inca.gov.br>. Acesso: em 2012.

[16] Marques Filho, O.; Vieira Neto, H. "Processamento Digital de Imagens". 1. ed. Rio de Janeiro: Brasport, 1999. 307 p.

[17] MEDED. J. L. Melton, \& MD, Editores. Skin Cancer and Benign Tumor Image - Loyola University - Chicago. Disponível em Loyola University Dermatology Medical Education: <http://www.meddean.luc.edu/lumen/MedEd/medicine/>. Acesso em 2011.

[18] Ministério da saúde; Instituto Nacional De Câncer. "Estimativa 2012: incidência de câncer no Brasil". Instituto Nacional de Câncer José Alencar Gomes da Silva, Coordenação Geral de Ações Estratégicas, Coordenação de Prevenção e Vigilância. Rio de Janeiro: INCA, 2011. 118 p. 
[19] Mumford, D. Shah, J. "Optimal approximations by piecewise smooth functions and associated variational problems". Communications on Pure and Applied Mathematics, v. XLII, p. 577-685, 1989.

[20] Nordström, K. N. "Biased anisotropic diffusion: a unified regularization and diffusion approach to edge detection". In: Proceedings of The First European Conference on Computer Vision, 1990, New York. p. 18-27.

[21] Norton, K. -A.; Iyatomi, H.; Celebi, M. E.; Schaefer, G.; Tanaka, M.; Ogawa, K. "Development of a novel border detection method for melanocytic and non-melanocytic dermoscopy images". In: Annual International Conference of the IEEE EMBS, 32nd. Buenos Aires, Argentina, 2010, p. 5403-5406.

[22] Osher, S.; Sethian, J. A. "Fronts propagating with curvature dependent speed: algorithms based on Hamilton-Jacobi formulations". Journal of Computational Physics, v. 79, p. 12-49, 1988.

[23] Otsu, N. "A threshold selection method from gray-level histograms". IEEE Transactions on Systems, Man, and Cybernetics, v. SMC-9, p. 62-66, 1979.

[24] Perona, P; Malik, "J. Scale-space and edge detection using anisotropic diffusion". IEEE transactions on pattern analysis and machine intelligence, v. 12, n. 7, p. 629-639, 1990.

[25] Zhang, N.; Zhang, J.; Shi, R. "An Improved Chan-Vese model for medical image segmentation". In: International Conference on Computer Science and Software Engineering, Wuhan, Hubei, 2008, p. 864-867.

[26] Zhao, J.; Shao, F.; Xu, Y.; Zhang, X.; Huang, W. "An improved Chan-Vese model without reinitialization for medical image segmentation". In: International Congress on Image and Signal Processing (CISP 2010), 3rd. Yantai, 2010, p. 1317-1321. 